\title{
EARLY DETECTION OF HELLP SYNDROME USING BIOLOGICAL MARKERS AND DOPPLER ULTRASONOGRAPHY
}

Al-Bokhary, M. S. ${ }^{(1)}$; Marei, Elham. S. ${ }^{(2)}$ and Al-Debaiky, Kh. F. ${ }^{(2)}$

1) Institute of Environmental Studies and Research Ain Shams University 2) National Center for Radiation Research and Technology (NCRRT), Atomic Energy Authority (AEA).

\begin{abstract}
Purpose: To determine the relationship between pregnancy-associated plasma protein-A (PAPP-A) levels and uterine Doppler ultrasonography for the prediction of severe preeclampsia (PE) and HELLP syndrome at the last trimester.

Methods: Two groups were constructed; severe preeclampsia group consisted of 38 women; HELLP Syndrome group consisted of 12 women. Both groups were matched strictly for gestational age at last trimester. Maternal blood sample for PAPP-A was collected and uterine Doppler ultrasonography was performed as soon as any woman under the study was diagnosed as severe preeclampsia or HELLP syndrome at last trimester and compared.

Results: Mean ages of participants, Parity, gestational weeks and fetal weights were similar. Mean PAPP-A levels were significantly higher in severe preeclampsia and HELLP groups. There was a statistically significant difference regarding Resistive Index $(\mathrm{RI})(\mathrm{P}<0.001)$ and Pulsatility Index $(\mathrm{PI})$ $(\mathrm{P}=0.005)$ between cases presenting with severe preeclampsia and HELLP groups.

Conclusion: PAPP-A levels could provide prediction for severe preeclampsia and HELLP syndrome at last trimester. Increased uterine artery resistance in the third trimester of pregnancy could be used to predict maternal complications including severe preeclampsia and HELLP syndrome.

Keywords: pre-eclampsia; HELLP syndrome; early prediction; maternal risk factors; mean maternal arterial pressure; Doppler ultrasound parameters; biomarker; pregnancy associated plasma protein-A (PAPP-A).
\end{abstract}


J. Environ. Sci.

Institute of Environmental Studies and Research - Ain Shams University

\section{INTRODUCTION}

HELLP was first described by Weinstein in 1982. The acronym is for haemolysis $(\mathrm{H})$, elevated liver enzymes (EL), thrombocytopenia (low platelet count LP). The name of the syndrome (hell + help) suggests the severity of maternal and fetal prognosis. It occurs in $0.17-0.85 \%$ of all pregnancies, and is more frequent in older multiparous Caucasian women. In $70 \%$ of the cases, the disorder is diagnosed antepartum: 10\% before 27 weeks of gestation (WG), 70\% between $27-37$ WG, $20 \%$ after 37 WG. In $30 \%$ of cases it is diagnosed postpartum since it has a poor prognosis (Siabi, 2004).

HELLP syndrome is a multisystemic disorder, whose etiopathogenesis is not completely understood. The most widely accepted hypotheses are: a change in the immune feto-maternal balance, platelet aggregation, endothelial dysfunction, arterial hypertension and an inborn error of the fatty acid oxidative metabolism. Hepatic involvement occurs by intravascular fibrin deposition and hypovolemia. Materno-fetal complications cause a 6.7-70\% perinatal mortality rate and a $1-24 \%$ maternal mortality rate. The recognition of HELLP syndrome and the rapid initiation of therapy are required for the improvement of materno-fetal prognosis (Holub et al., 2005).

Preeclampsia, which affects about 3 to $5 \%$ of pregnant women, is the most frequent medical complication in pregnancy and the most important cause of maternal and perinatal morbidity and mortality. During the past three decades, numerous clinical, biophysical, and biochemical screening tests have been proposed for the early detection of preeclampsia. Literature shows large discrepancies in the sensitivity and predictive value of several of these tests. 
No single screening test used for preeclampsia prediction has gained widespread acceptance into clinical practice. Instead, its value seems to be in increasing the predictive value of panels of tests, which include other clinical measurements (Hulstein et al., 2006).

Pregnancy-associated placental protein A (PAPP-A) is a large and highly glycosylated protein, which is produced by the developing trophoblast. PAPP-A modulates the activity of insulin-like growth factors by cleaving insulin-like growth factor binding proteins. It is proposed to play a role in implantation and is used as a biomarker for Down's syndrome. In chromosomally normal early pregnancies, there is evidence that low maternal serum PAPP-A is associated with an increased risk of subsequently developing PE. However, measurement of PAPP-A is not an effective stand alone screening tool for PE, because less than $20 \%$ of the affected cases present serum levels below the fifth centile (Gagnon et al., 2008).

Poor placentation with deficient remodeling of the spiral arteries has been associated with subsequent development of the early-onset forms of PE, Intra-Uterine Growth Restriction (IUGR), and other associated complications. In these abnormal pregnancies, the uteroplacental circulation remains in a state of high resistance, which can be measured noninvasively by uterine artery Doppler ultrasound. The impedance is increased in early PE and IUGR and predates the onset of the clinical symptoms by several weeks (Miller et al., 2008).

A number of studies examined the effectiveness of uterine artery Doppler in predicting the complications associated with impaired placentation. Early reports used subjective qualitative assessment evaluating the presence of a 
diastolic notch. However, in the last ten years, the use of continuous variables has achieved widespread acceptance as they provide more objective measurements to quantify the vascular impedance. Specifically, the mean pulsatility index (PI) has been studied as an objective measurement, and normal ranges from 11 to 41 weeks have been published (Gomez et al., 2008).

The aim of this study was to examine the combination of uterine artery Doppler study, together with PAPP-A measurement for the early prediction of the HELLP syndrome

\section{MATERIALS AND METHODS}

This prospective study was carried out at Al Galaa maternity teaching Hospital between August 2014 and September 2015. Two strictly, gestational age matched groups were constructed. The $1^{\text {st }}$ group consisted of 38 women who were referred to the hospital with the diagnosis of severe preeclampsia or attending the hospital and diagnosed as severe preeclampsia during routine prenatal visits at last trimester. The $2^{\text {nd }}$ group consists of 12 women who were referred to the hospital with the diagnosis of HELLP Syndrome or attending the hospital and diagnosed as HELLP Syndrome during routine prenatal visits at last trimester. Blood samples were taken and Uterine Doppler Ultrasonography were performed to the $1^{\text {st }}$ group during prenatal visits at last trimester of gestational weeks that match that of the $2^{\text {nd }}$ group and these women are followed up to delivery. Blood samples were taken and Uterine Doppler Ultrasonography was performed in both groups as soon as they were diagnosed as severe preeclampsia or HELLP Syndrome. If prenatal problems 
were diagnosed during examination such as presenting with labour pains, intra-uterine growth restriction or gestational diabetes, they were excluded from the group together with cases of multiple gestation.

History of chronic hypertension, diabetes mellitus, gestational diabetes, fetal demise or severely distressed at admission, gestational age $<26$ weeks, women in labor and/or with uterine activity, autoimmune diseases, clinical urgency with maternal hemodynamic instability or indication for immediate termination of pregnancy and other forms of pregnancy hypertension not classified as severe preeclampsia according to the present study criteria.

Preeclampsia was diagnosed when a blood pressure higher than 140/90 $\mathrm{mmHg}$ and proteinuria higher than $300 \mathrm{mg} / 24 \mathrm{~h}$ were observed on at least two occasions more than $6 \mathrm{~h}$ apart, after the 20th week of pregnancy. Preeclampsia were classified as severe if systolic blood pressure increased to at least $160 \mathrm{mmHg}$ and diastolic blood pressure to at least $110 \mathrm{mmHg}$ and proteinuria was $>5 \mathrm{~g}$ per day, in the presence of any combination of headache, visual disturbances, upper abdominal pain, oliguria $(<30 \mathrm{ml} / \mathrm{h})$, hyperbilirubinemia. elevated serum creatinine levels $(>0.8 \mathrm{mg} / \mathrm{dl}$. Thrombocytopenia $(<150.000 / \mathrm{mm} 3)$ and elevated aspartate or alanine aminotransferase levels.

Age of women, parity, gravidity, pregnancy outcomes, blood pressure values, neonatal weights, maternal weights and height were recorded. Body mass index were calculated. Participants were excluded if they had a pregnancy termination, a major fetal anomaly, a twin pregnancy, or if the pregnancy outcome was unknown (i.e. if they did not deliver in the hospital). Gestational age was determined from the date of the last menstrual period 
(L.M.P.). If this differed from the ultrasound estimate by more than 7 days, the ultrasound estimate was used. All participants signed an informed consent.

If hemolysis or elevated liver enzymes or a low platelet count was determined, patients were diagnosed as HELLP syndrome. The quantitative determination of PAPP-A in the maternal serum was performed with an Immulita/Immulite 1000 device using solid phase, chemiluminescence immunometric sandwich method. Blood samples were centrifugated as soon as they were taken; they were not stored in deep freeze and studied as soon as possible.

Statistical analysis: Statistical analysis was performed by SPSS 17.0 package program. Analyses of continuous variables of the groups were performed using multiple comparisons with Bonferroni correction. Correlations between PAPP-A, fetal weight and gestational age were investigated by Spearman test. Receiver operating curve (ROC) was performed between the two groups. $\mathrm{p}<0.05$ was accepted as significant at $95 \%$ confidence interval. The power of the study $(1-\beta)$ was found as $83.08 \%$. Regarding measuring in the data analysis of the uterine artery Doppler scans, the independent variable "uterine artery Doppler" was classified as normal or high-resistance Doppler. High-resistance Doppler was defined as the presence of bilateralarity diastolic notch and/or RI $>0.70$ in both arteries and/or PI above the $95^{\text {th }}$ percentile for the non-placental uterine artery, according to gestational age, in both arteries, as reported by Onwudiwe et al., 2008. 


\section{RESULTS}

Mean gestational ages of the severe PE and HELLP groups were 31.48 \pm 3.17 and 31.2 \pm 2.28 respectively. Demographic properties and estimated fetal weights by ultrasonography of the groups were similar (Table 1).

Table (1): Demographic: characteristics of the groups

\begin{tabular}{|c|c|c|}
\hline & Group1 (PE) (n=38) & Group2 (HELLP)(n=12) \\
\hline \hline $\begin{array}{c}\text { Age (year) } \\
\text { (weeks) }\end{array}$ & $26.2 \pm 4.8$ & $26.9 \pm 5.3$ \\
\hline Parity (number) & $2.1 \pm 1.2 \pm 3.17$ & $31.2 \pm 2.28$ \\
\hline Body Mass Index & $27.4 \pm 4.8$ & $2.3 \pm 1.3$ \\
\hline Estimated Fetal Weight & $1869 \pm 541$ & $28.3 \pm 5.3$ \\
\hline $\begin{array}{c}\text { Diastolic Blood pressure } \\
\text { (mmHg) }\end{array}$ & $118.4 \pm 6.3$ & $1821 \pm 604$ \\
\hline $\begin{array}{c}\text { Systolic Blood pressure } \\
\text { (mmHg) }\end{array}$ & $168.3 \pm 9.1$ & $157.6 \pm 8.1$ \\
\hline Urinary protein mg/24h & $3254 \pm 1121$ & $2627 \pm 1283$ \\
\hline PAPP-A (mIU/ml) & $89.2 \pm 11.2$ & $99.2 \pm 13.4$ \\
\hline
\end{tabular}

Mean 24 hours urinary protein excretion of group 1 was higher than group 2. Mean blood pressures (both systolic and diastolic) of the groups were not significantly different (Table 1). Mean PAPP-A levels were significantly higher in severe preeclampsia and HELLP groups than normal levels (normal level of PAPP-A: 2819 miu/L "1.00 MoM"). PAPP-A levels were not different when severe preeclampsia was compared with the group of HELLP syndrome (Table 1). There was a significantly positive and strong correlation between the gestational age and PAPP-A levels and also between fetal weight and PAPP-A levels (correlation coefficents $=0.83$ and 0.78 , respectively). 
Mean time interval between PAPP-A assessment and delivery was $3.51 \pm$ 2.15 days in severe-preeclampsia and $1.6 \pm 1.22$ days in HELLP syndrome. There was no significant correlation between PAPP-A levels and delivery time for both groups (correlation coefficent $=0.34$ ) $($ Table 2$)$.

Table (2): Mean time interval between PAPP-A assessment and delivery

\begin{tabular}{|c|c|c|}
\hline & Mean time interval (days) & correlation coefficent \\
\hline \hline Group 1 (PE) & $3.51 \pm 2.15$ & \multirow{2}{*}{0.34} \\
\hline Group 2 (HELLP) & $1.6 \pm 1.22$ & \\
\hline
\end{tabular}

With respect to uterine artery Doppler, in the severe preeclampsia group $(n=38)$; bilateral early diastolic notch was found in 22 cases $(57.9 \%)$. In another 10 patients $(26.3 \%)$, it was found unilaterally, while in $6(15.8 \%)$, early diastolic notch was not found in either of the uterine arteries (Table 3; Figure 1).

Table (3): Results of uterine artery Doppler early diastolic notch in patients with severe preeclampsia in the $2^{\text {nd }}$ half of pregnancy.

\begin{tabular}{|c|c|c|}
\hline Results of Doppler Evaluation & Number (38) & Percentage \\
\hline \hline Bilateral early diastolic notch & 22 & $57.9 \%$ \\
\hline Unilateral early diastolic notch & 10 & $26.3 \%$ \\
\hline Absent early diastolic notch & 6 & $15.8 \%$ \\
\hline
\end{tabular}




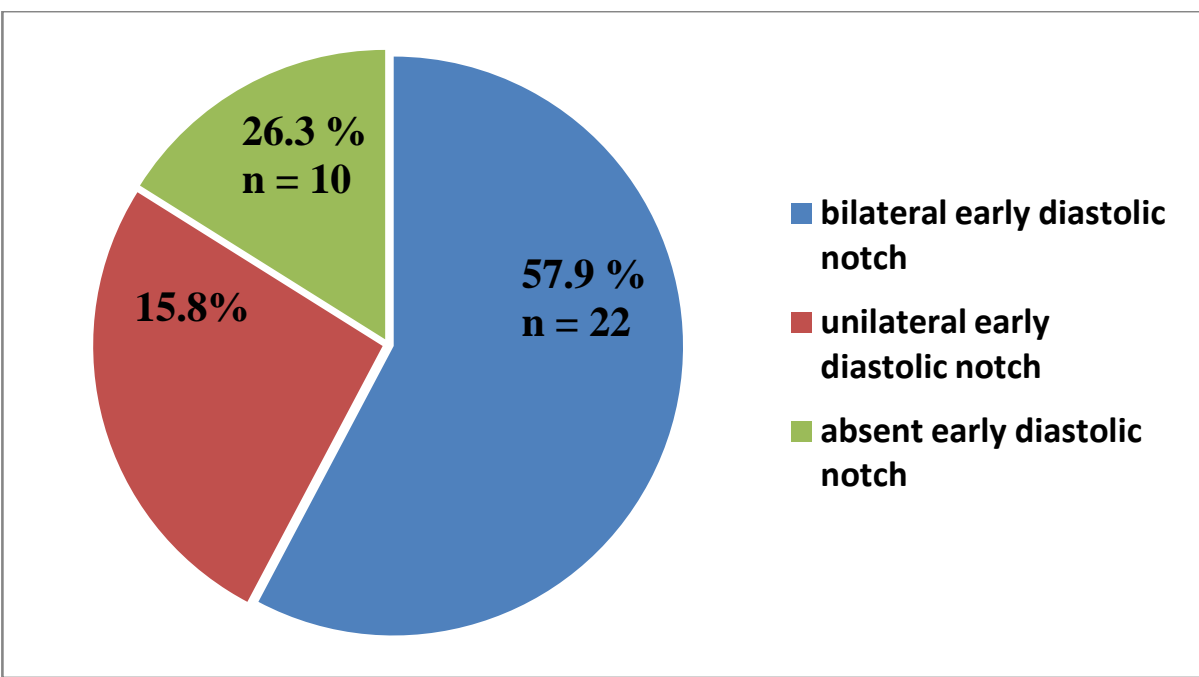

Figure (1): Results of uterine artery Doppler early diastolic notch in patients with severe preeclampsia in the $2^{\text {nd }}$ half of pregnancy.

The mean pulsatility index found was 1.38 for the right uterine artery and 1.36 for the left uterine artery. The mean resistance index was 0.60 in both arteries (Table 4; Figure 2).

Table (4): Results of uterine artery Doppler Pulsatility Index and Resistance Index (range and mean) in patients with both severe preeclampsia and HELLP syndrome in the $2^{\text {nd }}$ half of pregnancy.

\begin{tabular}{|c|c|c|}
\hline Pulsatility Index & Range & Mean \pm SD \\
\hline \hline Right uterine artery & $0.46-3.25$ & $1.38 \pm 0.60$ \\
\hline Left uterine artery & $0.57-3.30$ & $1.36 \pm 0.53$ \\
\hline Resistance Index & Range & Mean \pm SD \\
\hline Right uterine artery & $0.36-0.89$ & $0.60 \pm 0.12$ \\
\hline Left uterine artery & $0.36-0.95$ & $0.60 \pm 0.12$ \\
\hline
\end{tabular}


J. Environ. Sci.

Institute of Environmental Studies and Research - Ain Shams University

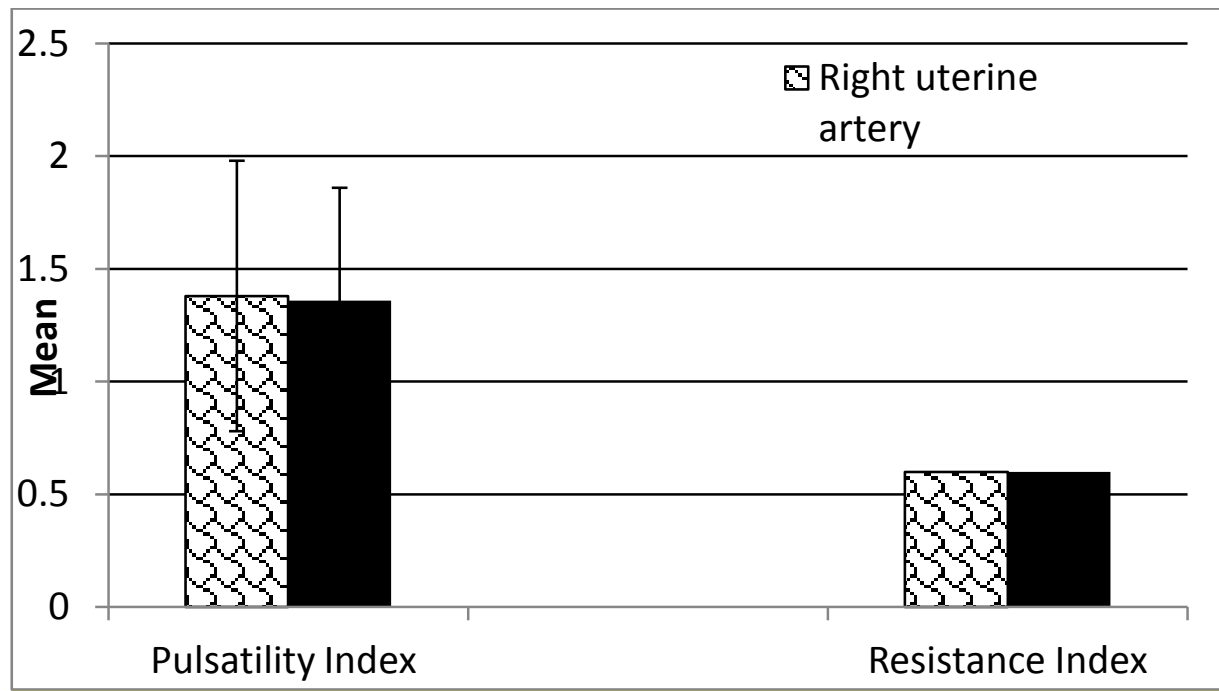

Figure(2): Results of uterine artery Doppler Pulsatility Index and Resistance Index (mean) in patients with both severe preeclampsia and HELLP syndrome in the $2^{\text {nd }}$ half of pregnancy.

While in the HELLP syndrome group $(n=12)$; bilateral early diastolic notch was found in 8 cases $(66.6 \%)$. In an another 2 patients $(16.7 \%)$, it was found unilaterally, while in the last 2 patients (16.7\%), early diastolic notch was not found in either of the uterine arteries (Table 5; Figure 3 ).

Table (5): Results of uterine artery Doppler early diastolic notch in patients with HELLP syndrome in the $2^{\text {nd }}$ half of pregnancy.

\begin{tabular}{|c|c|c|}
\hline Results of Doppler Evaluation & Number (12) & Percentage \\
\hline \hline Bilateral early diastolic notch & 8 & $66.6 \%$ \\
\hline Unilateral early diastolic notch & 2 & $16.7 \%$ \\
\hline Absent early diastolic notch & 2 & $16.7 \%$ \\
\hline
\end{tabular}




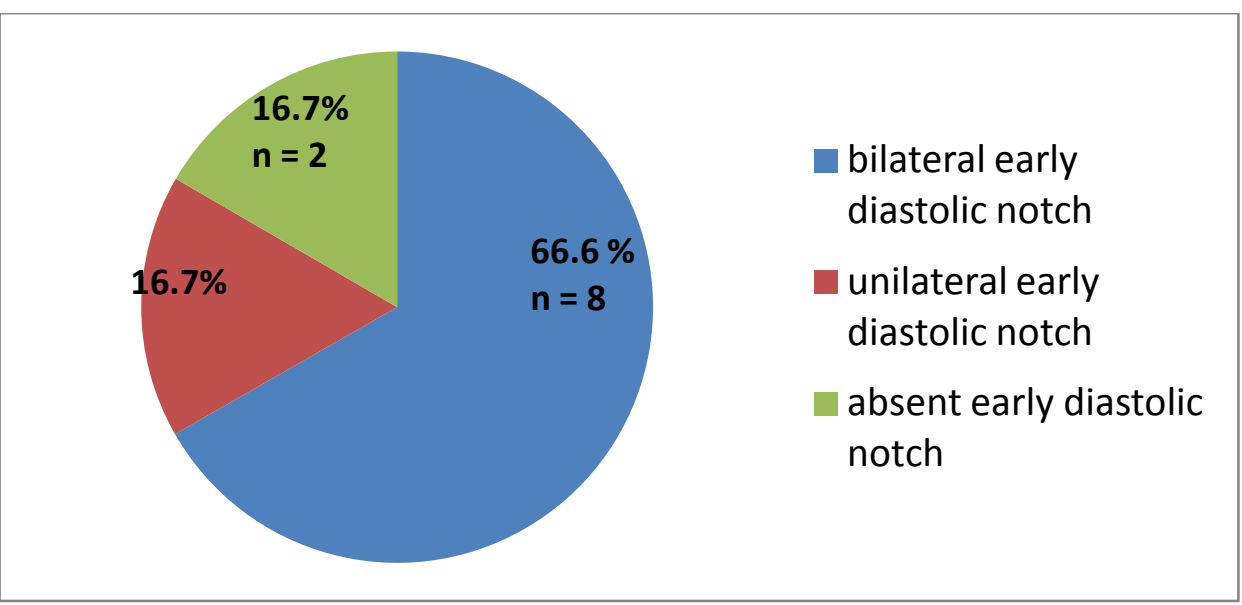

Figure (3): Results of uterine artery Doppler early diastolic notch in patients with HELLP syndrome in the $2^{\text {nd }}$ half of pregnancy.

Table (6): Results of uterine artery Doppler early diastolic notch in patients with both severe preeclampsia and HELLP syndrome in the $2^{\text {nd }}$ half of pregnancy.

\begin{tabular}{|c|c|c|}
\hline Doppler carried out in the mothers & Number (50) & Percentage \\
\hline \hline Bilateral early diastolic notch & 30 & $60 \%$ \\
\hline Unilateral early diastolic notch & 12 & $24 \%$ \\
\hline Absent early diastolic notch & 8 & $16 \%$ \\
\hline
\end{tabular}


J. Environ. Sci.

Institute of Environmental Studies and Research - Ain Shams University

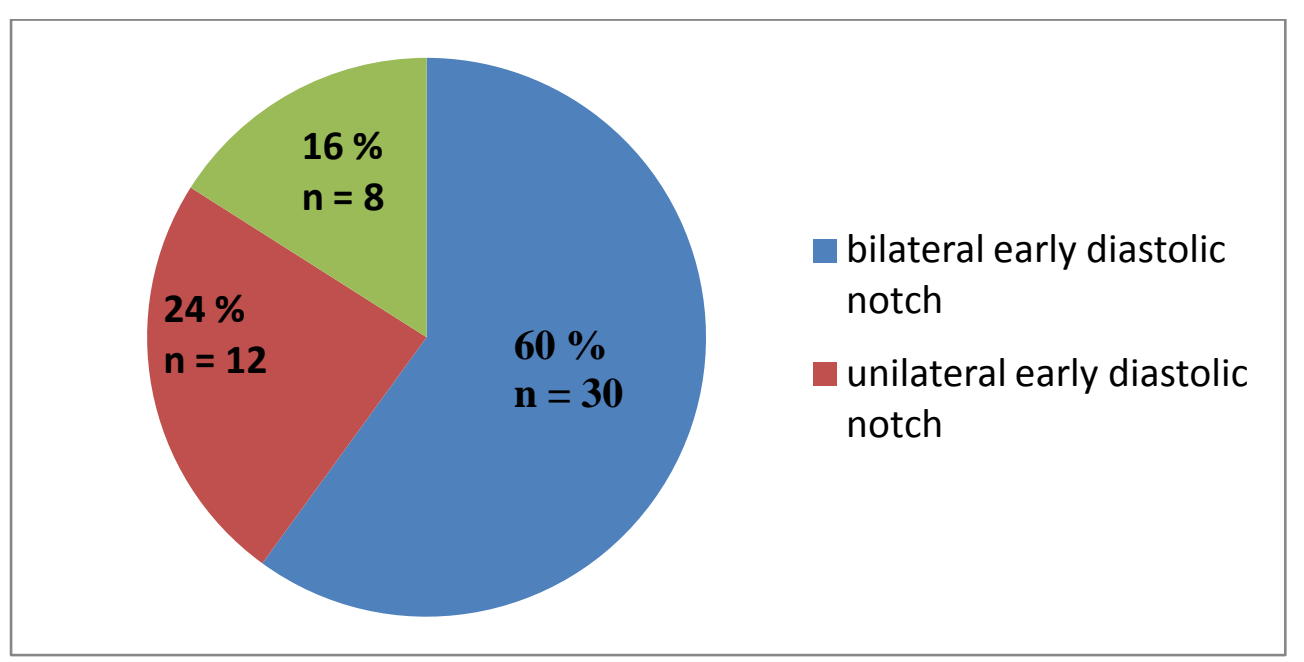

Figure (4): Results of uterine artery Doppler early diastolic notch in patients with both severe preeclampsia and HELLP syndrome in the $2^{\text {nd }}$ half of pregnancy.

\section{DISCUSSION}

The etiology of preeclampsia is still unknown but it is now clear that this condition is characterized by poor implantation and development of the trophoblast in the maternal decidua (Davison et al., 2004). However, although the diagnosis of severe preeclampsia is readily achievable by current clinical assessments, clinicians are awaiting the development of biomarkers that can be used during the early phases of apparently uncomplicated pregnancy to predict the subsequent development of preeclampsia. To date, several proteins that are elevated in the maternal sera, including activin A/ inhibin A (Canini et al., 2008), leptin (Davison et al., 2004), soluble Fms-related tyrosine kinase 
(FLT1) (Deveci et al., 2009) and endoglin (Davison et al., 2004) have been suggested as possible biological markers of preeclampsia.

More recent studies have shown that reduced first trimester serum levels of PAPP-A are associated with subsequent preeclampsia (Davison et al., 2004). It has been suggested that PAPP-A is useful as a marker of fetal growth restriction at the first trimester (Davison et al., 2004) but there are not many studies concerning late pregnancy levels of PAPP-A at preeclampsia.

PAPP-A was first shown to be elevated in the plasma of preeclamptic women nearly 30 years ago. It was showed that elevated levels in severe preeclampsia were detected within 24 hours of admission to hospital but PAPP-A values in mild-preeclampsia did not differ significantly from the normal value for the corresponding stage of gestation (Nishizawa et al., 2008). Some of the later studies confirmed this post onset elevation of serum PAPP-A (Davison et al., 2004). But some other studies reported that there is no correlation of PAPP-A in preeclampsia but PAPP-A2 instead (Deveci et al., 2009). Uncertainty of specificity of the anti-PAPP-A antibodies possibly underlies this discrepancy. PAPP-A2 was the first protein identified as having a similar structure to PAPP-A (Davison et al., 2004). Both PAPP-A and -A2 are involved in the IGF pathway. PAPP-A is a protease that cleaves IGFbinding protein IGFBP4 and IGFBP5, leading to the activation of IGF-I and II, whereas PAPP-A2 has been reported to cleave only IGFBP5 (Davison et al., 2004). Both enzymes are expressed in a wide range of tissues, but abundantly in placental syncytiotrophoblasts and the pregnant uterus. 
Nishizawa et al. (2008) reported that instead of PAPP-A, PAPP-A2 increased in placenta of preeclamptic women compared to uncomplicated pregnancy by western blot and quantitative RT-PCR analysis. They also found that, serum PAPP-A2 levels were detected at significantly higher levels in patients with preeclampsia, the pre-eclampsia cut off value of $39.32 \mathrm{ng} / \mu \mathrm{l}$ allowed for both high sensitivity (95.0\%) and high specificity $(85.7 \%)$. However, the serum concentrations of PAPP-A did not correlate well with the presence of preeclampsia.

Bersinger et al. (2003) have measured the concentrations of PAPP-A in placental extracts to specifically study the changes in the placenta in preeclampsia as serum levels reflect cumulative changes in all potential sources. They found that the placental content reflected the serum pattern for PAPP-A in preeclampsia, suggesting that the placenta is a source of the elevated circulating proteins in preeclampsia.

Our study is consistent with Bersingefls and Deveci's studies that PAPPA at last trimester significantly increases in severe preeclampsia and HELLP syndrome (Davison et al., 2004). Deveci et al. (2009) also found positive correlation between PAPP-A level and mean blood pressure of the preeclamptic women and concluded that level of PAPP-A is related with severity of the disease.

The level of blood pressure is only one of the factors representing the severity of preeclampsia. After the cut off value of $160 / 110 \mathrm{mmHg}$ which discriminates the mild and severe preeclampsia, there is no enough finding to conclude that more increase in blood pressure means more severe the disease 
(Canini et al., 2008).That is why, instead of performing correlation analysis between mean blood pressure with PAPP-A levels, we preferred grouping the women as severe preeclampsia and HELLP syndrome according to classical worldwide criteria. We found that PAPP-A at last trimester increased in both severe preeclampsia and HELLP syndrome and there was no significant difference in PAPP-A levels between the two groups which indicate that PAPP-A level is not related to severity of the disease.

Mean uterine artery Doppler measurements (pulsatility and resistance indices) found in the women evaluated in this study (1.37 and 0.60, respectively) may be considered high, as some investigators consider pulsatility indices $(\mathrm{PI})>1.20$ and resistance indices $(\mathrm{RI})>0.58$ as resistant (Schwarze et al., 2005). This result may be explained by the fact that only women in whom diagnosis of preeclampsia had already been defined were included in this study. With respect to the frequency of persistence of early diastolic notch, bilateral in $60 \%$ of cases, unilateral in $24 \%$ and both bilateral and unilateral in $84 \%$, these values are higher than those reported in a study carried out in the third trimester of pregnancy in patients with preeclampsia in which the frequency of persistence of early diastolic notch was reported as $35 \%$ (Li et al., 2005). These findings suggest a significant vasospastic pattern in the women in this study that may be explained by the strictness of the admission criteria adopted for participation in the trial.

With respect to the evaluation of the biological characteristics (age and BMI) of the patients enrolled in the present study, the results found were similar to those reported in other studies carried out in the northeast of Brazil (Melo et al., 2007); however, the mean age of 26 years of patients in the 
present study was lower than the mean of 32 years reported in European studies on preeclampsia (Axt-Fliedner et al., 2005). Considering that maternal age is an indicator of the level of development of a country (Save the Children. Sate of the World's Mothers (2007), this finding corresponds to what would be expected for the local population of a developing country. With respect to BMI, the mean of $27.8 \mathrm{~kg} / \mathrm{m} 2$ found in this study is high and indicative of overweight, and is similar to other reports on patients with preeclampsia (Plasencia et al., 2007, Papageorghiou et al., 2005). There was no statistically significant difference between the groups with respect to age, parity or BMI. Therefore, the characteristics of the majority of primiparous patients with a tendency towards overweight found in the present study are similar to the profile observed in patients with a greater risk for preeclampsia in pregnancy (Duckitt et al., 2005).

The present study also has certain limitations. Collection of serum for PAPP-A for every gestational week for every patient may be considered as a first limitation of the study because it is known that PAPP-A levels increase throughout the pregnancy so the groups must be strictly age matched. Another limitation is relatively small number of participants especially for HELLP group. Further studies involving large samples at last trimester are needed.

\section{CONCLUSION}

The results of this study provide further support to the hypothesis that PAPP-A levels at late pregnancy increase in severe preeclampsia and HELLP syndrome. A cutoff level could be useful for differentiating normal pregnancy 
from preeclampsia. The level of PAPP-A is strongly correlated with fetal birth weight and gestational age but not associated with severity of the disease.

The introduction of Doppler technology has provided the first opportunity for repetitive non invasive hemodynamic monitoring in human pregnancy. There is ample evidence that Doppler indices from the fetomaternal circulation can reliably predict adverse perinatal outcome in an obstetric patient population with a high prevalence of severe preeclampsia and HELLP syndrome. Compared to other methods of fetal monitoring Doppler has proved to be more sensitive in detecting fetal compromises early.

Doppler investigation of the feto-maternal circulation play an important role in monitoring high risk pregnancies and thereby help to determine the optimal time for delivery. Hence, the use of Doppler provides information that is not readily obtained from more conventional tests of fetal well being. It therefore has an important role to play in the management of high risk pregnancies.

\section{RECOMMENDATION}

It is possible that future studies with an adequate sample size will show statistically significant differences with respect to the frequency of these outcomes.

There is a clear need to increase the number of studies in this area given the growing literature relating hypertensive disorders of pregnancy to future cardiovascular disease. For a more precise evaluation of this association, knowledge on the clinical evolution of these patients in the puerperium is essential. 
With respect to uterine artery Doppler evaluation, the objective of this study and the majority of previous studies have been to predict the disease not the adverse obstetrical outcomes and not to evaluate complications in the postpartum so future studies should be directed towards possible post partum complications. Future studies with larger sample sizes, calculated according to the rate of each complication, are necessary in order to test the hypothesis of an increased risk of intra and postpartum complications in women with an abnormal uterine artery Doppler scan in pregnancy.

\section{REFERENCES}

Axt-Fliedner R, Schwarze A, Nelles I, Altgassen C, Friedrich M and Schmidt W (2005): The value of uterine artery Doppler ultrasound in the prediction of severe complications in a risk population. Arch Gynecol Obstet ; 271:53-58.

Bersinger NA, Snuiraiam AK; Muttukrishna S.; Groome NP and Redman CW; (2003): Women with preeclampsia have increased serum levels of pregnancy-associated plasma protein A PAPP-A), A, activin A and soluble E-selevtin. Hypertens Pregnancy 22:45-55

Canini S., Prefumo F and Pastorino D; (2008): Association between birth weight and first trimester free beta-human chorionic gonadotropin and pregnancy-associated plasma protein A. Fert Steril 89:174178

Davison, liumuth V. and Jeyabalan A (2004): New aspects in the pathophysiology of Preeclampsia. Am Soc Nephrol 15:24402448.

Deveci K, Sogut E; Evliyaoglu 0 and Duras N; (2009): Pregnancy-associated plasma protein-A and C-reactive protein levels in pre-eclamptic and normotensive pregnant women at third trimester, J Obstet Gynaecol Res.2009, 35, 94-98. 
Duckitt K and Harrington D (2005): Risk factors for pre-eclampsia at antenatal booking: systematic review of controlled studies. BMJ; 330:565.

Gagnon A, Wilson RD; Audibert F; Allen VM; Blight C and Brock JA; (2008): Obstetrical complications associated with abnormal maternal serum markers analytes. J Obstet Gynaecol Can; 30(10):918-49.

Gomez O, Figueras F; Fernandez S; Bennasar M; Martinez JM and Puerto B; (2008): Reference ranges for uterine artery mean pulsatility index at 11-41 weeks of gestation. Ultrasound Obstet Gynecol.

Holub M, Bodamer OA; Item C; Mühl A; Pollak A and Stöckler- Ipsiroglu S; (2005): Lack of correlation between fatty acid oxidation disorders and haemolysis, elevated liver enzymes, low platelets (HELLP) syndrome? Acta Paediatr; 94: 48-52.

Hulstein JJ, van Runnard Heimel PJ and Franx A; (2006): Acute activation of the endothelium results in increased levels of active von Willebrand factor in hemolysis, elevated liver enzymes and low platelets (HELLP) syndrome. J Thromb Haemost; 4: 2569-2575.

Li H, Gudnason H, Olofsson P, Dubiel M and Gudmundsson S (2005): Increased uterine artery vascular impedance is related to adverse outcome of pregnancy but is present in only one-third of late third-trimester pre-eclamptic women. Ultrasound Obstet Gynecol; 25:459-463.

Melo ASO, Assunção PL, Gondim SSR, Carvalho DF, Amorim MMR and Benicio MHDA (2007): Estado nutricional materno, ganho de peso gestacional e peso ao nascer. Rev Bras Epidemiol; 10:249257.

Miller J, Turan S; Baschat AA; (2008): Fetal growth restriction. Seminars in Perinatology, Volume 32, Issue 4, pages 274-80.

Nishizawa, Pryor-Koishi K; Suzuki M. Kato T; Kogo H; Sekiya T; Kuraliashi $\mathrm{H}$ and Udagawa $\mathrm{Y}$ (2008): Increased levels of preg-nancyassociated nlrisms nrofein-47 in the kororn eclamptic patients. $\mathrm{Mu}$ ] Hum Reprod 14:595-602. 
Onwudiwe, N.; Yu, C.K.; Poon, L.C.; Spiliopoulos, I. and Nicolaides, K.H. (2008): Prediction of pre-eclampsia by a combination of maternal history, uterine artery Doppler and mean arterial pressure. Ultrasound Obstet. Gynecol., 32, 877-883.

Papageorghiou AT, Yu CK, Erasmus IE, Cuckle HS and Nicolaides KH (2005): Assessment of risk for the development of pre-eclampsia by maternal characteristics and uterine artery Doppler. BJOG; 112:703-709.

Plasencia W, Maiz N, Bonino S, Kaihura C and Nicolaides KH (2007): Uterine artery Doppler at $11+0$ to $13+6$ weeks in the prediction of pre-eclampsia. Ultrasound Obstet Gynecol; 30:742-749.

Save the Children. Sate of the World's Mothers (2007): May 2007. URL: http://www.savethechildren.org/publications/mothers/2007/SOW M-2007-final.pdf

Schwarze A, Nelles I, Krapp M, Friedrich M, Schmidt W and Diedrich K, (2005): Doppler ultrasound of the uterine artery in the prediction of severe complications during low-risk pregnancies. Arch Gynecol Obstet; 271:46-52.

Sibai BM, (2004): Diagnosis, controversies, and management of the syndrome of hemolysis, elevated liver enzymes, and low platelet count. Obstet Gynecol; 103: 981-991. 


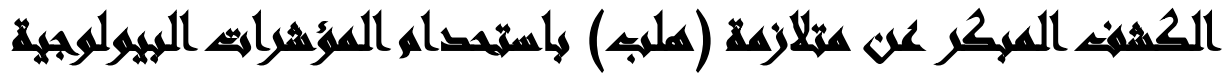

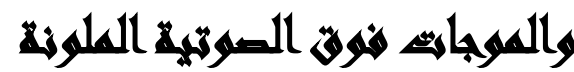

\section{$[r]$}

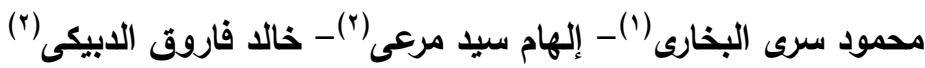

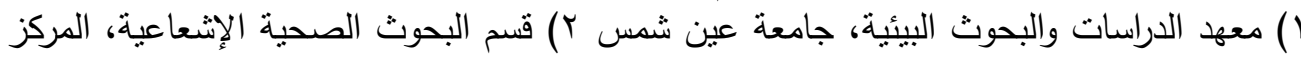
القومي لبحوث وتكنولوجيا الإشعاع، هيئة الطاقة الذربة الذية

\section{المستخطي}

متلازمة (هلب) تعنى اضطراب لعديد من أجهزة الجسم البشرى، غير المفهوم أسبابها المرضية.

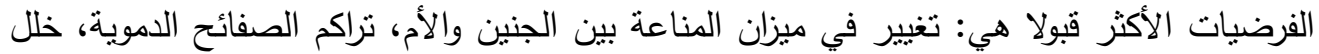

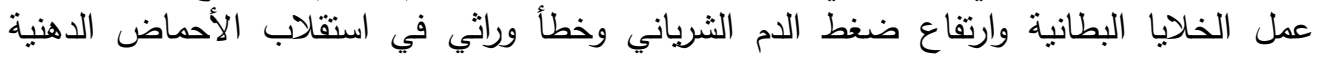

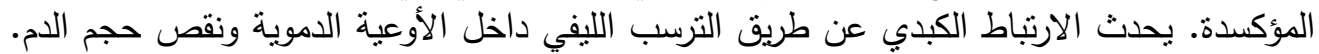

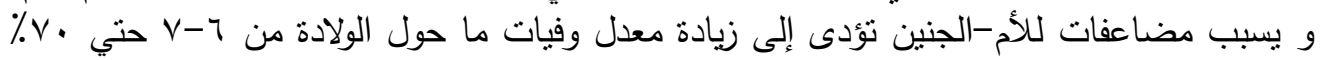

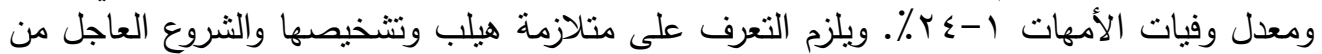

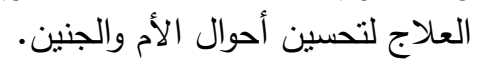

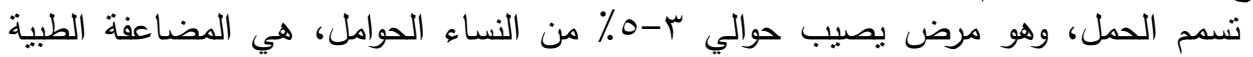

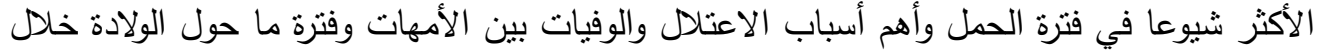

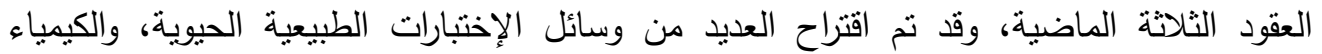

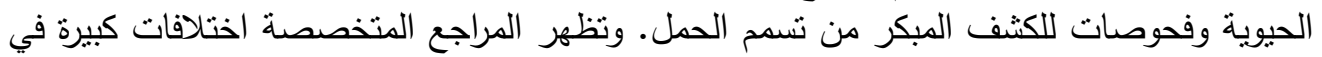

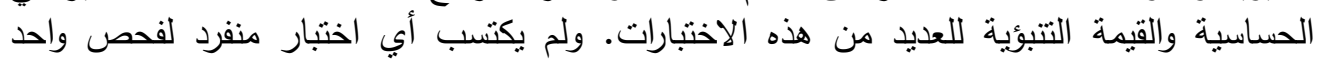

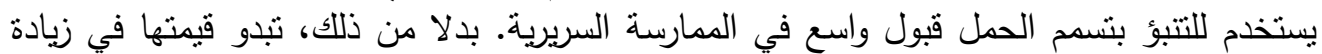

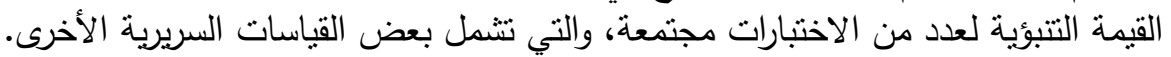

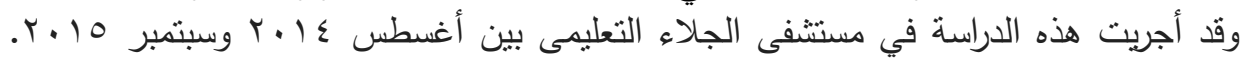

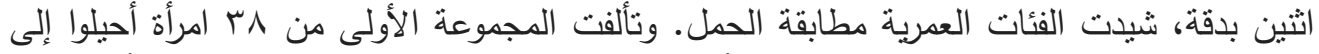

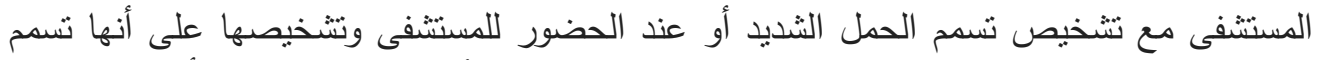

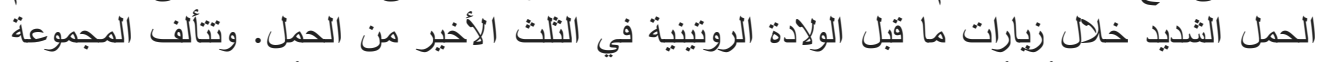

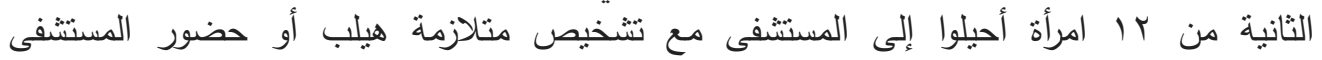

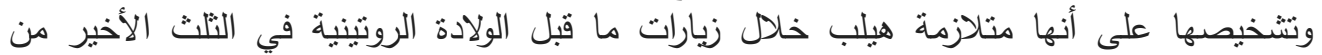

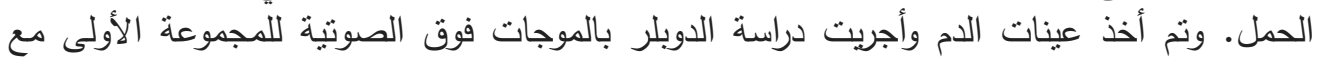

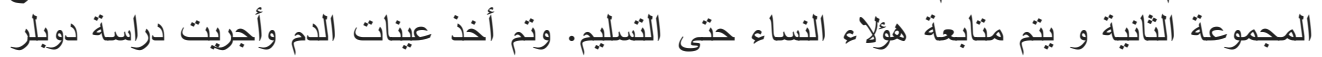
بالموجات فوق الصوتية في كل من المجموعتين في أقرب وقت ونت عندما تم تشخيصها على على أنها تسمح الحمل الثنديد أو متلازمة هلب. 
J. Environ. Sci.

Institute of Environmental Studies and Research - Ain Shams University

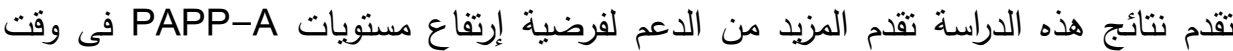

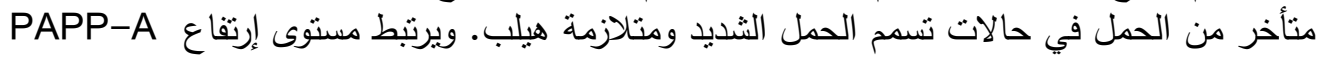

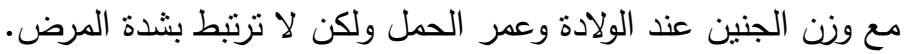

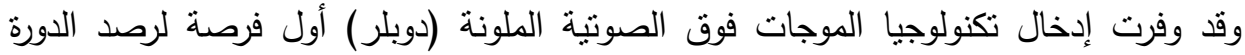

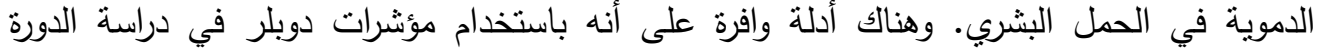

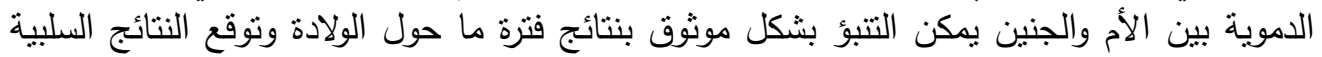

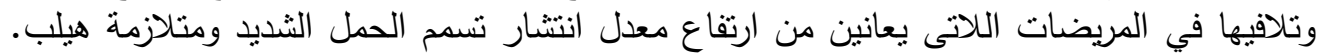

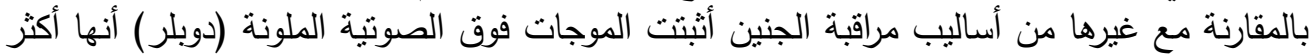

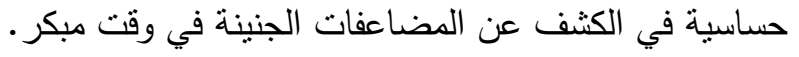

